\title{
RESEARCH
}

Open Access

\section{Mesial temporal lobe epilepsy with psychiatric comorbidities: a place for differential neuroinflammatory interplay}

Ludmyla Kandratavicius $^{1,2+}$, Jose Eduardo Peixoto-Santos ${ }^{1 \dagger}$, Mariana Raquel Monteiro ${ }^{1}$, Renata Caldo Scandiuzzi ${ }^{1}$, Carlos Gilberto Carlotti Jr ${ }^{3}$, Joao Alberto Assirati $\mathrm{Jr}^{3}$, Jaime Eduardo Hallak ${ }^{1,2,4}$ and Joao Pereira Leite ${ }^{1,2^{*}}$

\begin{abstract}
Background: Despite the strong association between epilepsy and psychiatric comorbidities, few biological substrates are currently described. We have previously reported neuropathological alterations in mesial temporal lobe epilepsy (MTLE) patients with major depression and psychosis that suggest a morphological and neurochemical basis for psychopathological symptoms. Neuroinflammatory-related structures and molecules might be part of the altered neurochemical milieu underlying the association between epilepsy and psychiatric comorbidities, and such features have not been previously investigated in humans.
\end{abstract}

Methods: MTLE hippocampi of subjects without psychiatric history (MTLEW), MTLE + major depression (MTLE + D), and MTLE + interictal psychosis (MTLE + P) derived from epilepsy surgery and control necropsies were investigated for reactive astrocytes (glial fibrillary acidic protein (GFAP)), activated microglia (human leukocyte antigen, MHC class II (HLA-DR)), glial metallothionein-I/II (MT-I/II), and aquaporin 4 (AQP4) immunohistochemistry.

Results: We found an increased GFAP immunoreactive area in the molecular layers, granule cell layer, and cornus ammonis region 2 (CA2) and cornus ammonis region 1 (CA1) of MTLE and MTLE + $\mathrm{P}$, respectively, compared to $M T L E+D$. HLA-DR immunoreactive area was higher in cornus ammonis region 3 (CA3) of MTLE + P, compared to $M T L E+D$ and MTLE $E_{W}$ and in the hilus, when compared to MTLE $E_{W}$. MTLE $E_{W}$ cases showed increased MT-I/II area in the granule cell layer and CA1, compared to MTLE + P, and in the parasubiculum, when compared to MTLE + D and MTLE + P. Differences between MTLE and control, such as astrogliosis, microgliosis, increased MT-I/II, and decreased perivascular AQP4 in the epileptogenic hippocampus, were in agreement to what is currently described in the literature.

Conclusions: Neuroinflammatory-related molecules in MTLE hippocampus show a distinct pattern of expression when patients present with a comorbid psychiatric diagnosis, similar to what is found in the pure forms of schizophrenia and major depression. Future studies focusing on inflammatory characteristics of MTLE with psychiatric comorbidities might help in the design of better therapeutic strategies.

Keywords: Temporal lobe epilepsy, Psychosis, Major depression, Astrocytes, Microglia, Aquaporin 4, Metallothionein

\footnotetext{
* Correspondence: jpleite@fmrp.usp.br

${ }^{\dagger}$ Equal contributors

${ }^{1}$ Department of Neurosciences and Behavior, Ribeirao Preto Medical School, University of Sao Paulo (USP), Av Bandeirantes 3900, CEP 14049-900 Ribeirao Preto, SP, Brazil

${ }^{2}$ Center for Interdisciplinary Research on Applied Neurosciences (NAPNA), USP, Ribeirao Preto, Brazil

Full list of author information is available at the end of the article
}

\section{Biomed Central}

(c) 2015 Kandratavicius et al.; licensee BioMed Central. This is an Open Access article distributed under the terms of the Creative Commons Attribution License (http://creativecommons.org/licenses/by/4.0), which permits unrestricted use, distribution, and reproduction in any medium, provided the original work is properly credited. The Creative Commons Public Domain Dedication waiver (http://creativecommons.org/publicdomain/zero/1.0/) applies to the data made available in this article, unless otherwise stated. 


\section{Background}

Mesial temporal lobe epilepsy (MTLE) is the most common cause of intractable epilepsy in adults and is characterized by hippocampal sclerosis, neuronal loss, gliosis, and mossy fiber sprouting [1-4]. Psychiatric comorbidities are frequent in MTLE patients, and in population-based studies, epilepsy has been consistently associated with increased risk of schizophrenia [5]. However, the exact biological substrate behind the association of MTLE and psychiatric comorbidities is unknown [6,7]. We have recently shown neuropathological alterations in the hippocampus of patients with epilepsy and the history of major depression or interictal psychosis, which may indicate that structural changes and neurochemical dysfunctions may underlie psychiatric symptoms in MTLE [8-10].

Neuroinflammation-related abnormalities such as glial pathology, glutamate dysregulation, and bloodbrain-barrier dysfunction are found not only in epilepsy, but also in schizophrenia and major depression [11]. Glial proteins, such as metallothionein I and II (MT-I/II), are able to quench free zinc and modulate glutamatergic neurotransmission [12], and aquaporin 4 (AQP4), found in astrocytic endfeets, is a regulator of water homeostasis that majorly controls edema formation and tissue excitability $[13,14]$. In schizophrenia, upregulation of MT-I/II and of astrocyte and microglia markers have been documented in several brain regions [15-17]. By contrast, neuropathological studies in specimens from major depression patients indicate reduction in hippocampal glial fibrillary acidic protein (GFAP)-positive astrocytes and of AQP4 and MT-I/II in the frontal cortex $[18,19]$. Protein expression and neuropathological features in MTLE with psychiatric comorbidities may resemble what is found in the pure form of the correspondent psychiatric illness [20]. Therefore, we hypothesized that expression of reactive astrocytes, activated microglia, glial MT-I/II, and AQP4 would be altered in the hippocampal formation of MTLE patients with major depression and interictal psychosis.

\section{Methods}

\section{Patients}

We analyzed the hippocampal formation from MTLE specimens freshly collected in the operating room and non-epileptic controls from necropsy, collected between 4 and $9 \mathrm{~h}$ after death. A <24-h postmortem time limit allows comparison of necropsy tissue with freshly collected surgical specimens for their protein levels, cell morphology, and tissue integrity $[4,21,22]$. Tissue collection and processing were conducted according to a protocol approved by our institution's Research Ethics Board (\# 2634/2008 and \# 9370/2003).
MTLE specimens were derived from 43 MTLE patients who underwent a standard en bloc anterior temporal resection (including 3 to $4 \mathrm{~cm}$ of the hippocampus) for medically intractable seizures. All had clinical neuropathological confirmation of hippocampal sclerosis (HS). They were divided into three groups: 17 MTLE patients without any history of psychiatric disorder (MTLE $_{\mathrm{W}}$ group), 11 MTLE patients with interictal psychosis (MTLE + P group), and 15 MTLE patients with a diagnosis of major depression (MTLE $+\mathrm{D}$ group). For comparison purposes, 14 human non-epileptic control hippocampi from necropsies were processed and analyzed in the same manner as the surgical cases. Underlying diseases causing death were cardiomyopathy, sepsis, acute lymphoblastic leukemia, gastric adenocarcinoma, pulmonary infarct, or renal-hepatic failure, and patients had no history of hypoxic episodes during agony, seizures, or neurological diseases. Furthermore, there was no evidence of brain pathological abnormalities on clinical postmortem examination of the mesial temporal structures. MTLE and control specimens were collected between 1998 and 2008. A summary of clinical characteristics of all groups is depicted in Table 1.

\section{Clinical features of MTLE patients}

All patients were referred for pre-surgical assessment due to drug-resistant seizures as defined in the literature [23]. Patients were evaluated at the Ribeirao Preto Epilepsy Surgery Program using standardized protocols approved by the institution's Ethics Committee and a written consent form was obtained from each patient. Presurgical investigation at the Epilepsy Monitoring Unit included detailed clinical history, neurological examination, interictal and ictal scalp/sphenoidal electroencephalography (EEG), neuropsychology evaluation, and intracarotid amobarbital procedure (Wada test) for memory and language lateralization whenever deemed clinically necessary.

Definition of MTLE followed Engel's criteria [24]: (I) seizure semiology consistent with MTLE, usually with epigastric/autonomic/psychic auras, followed by complex partial seizures; (II) pre-surgical investigation confirming the seizure onset zone in the temporal lobe; (III) anterior and mesial temporal interictal spikes on EEG; (IV) no lesions other than uni- or bilateral hippocampal atrophy on high-resolution magnetic resonance imaging scans (reduced hippocampal dimensions and increased $\mathrm{T} 2$ signal); (V) clinical histopathological examination compatible with HS; and (VI) no evidence of dual pathology identifiable by any of the assessment methods described (clinical, electrophysiology, neuroimaging, and histopathology). Exclusion criteria were as follows: (I) focal neurological abnormalities on physical examination, (II) generalized or extra-temporal EEG spikes, and (III) marked cognitive impairment indicating dysfunction 
Table 1 Demographic and clinical data

\begin{tabular}{|c|c|c|c|c|c|}
\hline & MTLE $_{\mathrm{w}}$ & MTLE + D & MTLE + P & Controls & Statistics \\
\hline Male $(n)$ & 10 & 5 & 8 & 11 & No difference \\
\hline Female $(n)$ & 7 & 10 & 3 & 3 & \\
\hline IPI present $(n)$ & 7 & 10 & 8 & n.a. & No difference \\
\hline IPI absent $(n)$ & 10 & 5 & 3 & n.a. & \\
\hline Age of first seizure (years) & $3.8 \pm 3.3$ & $6.3 \pm 7.5$ & $7.4 \pm 8.5$ & n.a. & No difference \\
\hline Age when seizures became recurrent or age of onset (years) & $10.0 \pm 5.4$ & $12.0 \pm 9.7$ & $13.5 \pm 7.7$ & n.a. & No difference \\
\hline Seizure type: CPS $(n)$ & 7 & 9 & 5 & n.a. & No difference \\
\hline Seizure type: SGS $(n)$ & 10 & 6 & 6 & n.a. & \\
\hline Seizure frequency (monthly) & $14.3 \pm 11.4$ & $12.6 \pm 8.9$ & $16.4 \pm 11.2$ & n.a. & No difference \\
\hline Right HS (n) & 12 & 9 & 5 & n.a. & No difference \\
\hline Left HS (n) & 5 & 4 & 5 & n.a. & \\
\hline Bilateral HS (n) & 0 & 2 & 1 & n.a. & \\
\hline Right handedness $(n)$ & 14 & 14 & 11 & n.a. & No difference \\
\hline Left handedness $(n)$ & 2 & 0 & 0 & n.a. & \\
\hline Bilateral handedness ( $n$ ) & 1 & 1 & 0 & n.a. & \\
\hline Memory in verbal tasks: average or above $(n)$ & 7 & 7 & 2 & n.a. & No difference \\
\hline Memory in verbal tasks: below average $(n)$ & 10 & 8 & 9 & n.a. & \\
\hline Memory in non-verbal tasks: average or above $(n)$ & 11 & 9 & 3 & n.a. & No difference \\
\hline Memory in non-verbal tasks: below average $(n)$ & 6 & 6 & 8 & n.a. & \\
\hline Full-scale IQ & $85.9 \pm 7.7$ & $84.9 \pm 9.3$ & $83.4 \pm 7.1$ & n.a. & No difference \\
\hline Years at school & $7.1 \pm 3.5$ & $5.5 \pm 3.5$ & $6.3 \pm 4.1$ & n.a. & No difference \\
\hline Age at surgery (or at death for controls) (years) & $33.5 \pm 8.0$ & $37.6 \pm 11.7$ & $40.0 \pm 5.9$ & $42.6 \pm 16.0$ & No difference \\
\hline Duration of epilepsy (years) & $23.9 \pm 8.2$ & $24.9 \pm 13.6$ & $26.4 \pm 8.6$ & n.a. & No difference \\
\hline Collected side: right $(n)$ & 12 & 11 & 6 & 6 & No difference \\
\hline Collected side: left $(n)$ & 5 & 4 & 5 & 8 & \\
\hline Surgical outcome: complete remission ( $n$ ) & 15 & 10 & 9 & n.a. & No difference \\
\hline Surgical outcome: only auras and/or fewer seizures $(n)$ & 2 & 5 & 2 & n.a. & \\
\hline
\end{tabular}

Values indicated as mean \pm standard deviation when applicable. CPS: Complex partial seizure; HS: Hippocampal sclerosis; IPI: Initial precipitant injury; MTLE: mesial temporal lobe epilepsy; MTLE + D: MTLE + major depression; MTLE + P: MTLE + interictal psychosis; MTLE history; n.a.: Not applicable; SGS: Secondarily generalized seizures.

beyond the temporal regions (intelligence quotient (IQ) <69).

Information regarding antecedent of an initial precipitant injury, febrile seizures, seizure types, drug regimen, and estimated monthly seizure frequency (within the 2 years prior to surgery) were retrospectively collected from medical records for each patient. Psychiatric evaluations were conducted in all MTLE patients. Each diagnosis of major depression was independently established during the presurgical evaluation by two psychiatrists with experience in psychiatric disorders associated with epilepsy, using the guidelines of the Diagnostic and Statistical Manual of Mental Disorders, 4th edition. Once a consensus on the classification of psychotic syndromes associated with epilepsy was lacking at the time of collection, and neither DSM-IV nor ICD-10 has addressed this issue specifically (for a review, please see
[20]), the diagnosis of psychosis associated with MTLE was established according to Sachdev [25], meaning that patients with interictal psychosis did not experience the following: psychotic disorder temporally associated with seizures, changes in antiepileptic medications, epileptic status, delirium, and psychosis for paradoxical normalization (for review, please see [26]). This group was defined by a prolonged psychotic state that was not related to the epileptic seizures. Typically, the psychotic states closely resemble schizophrenia, with paranoid ideas which might become systematized, ideas of influence, and auditory hallucinations often of a menacing quality. The points of difference are as follows: common religious coloring of the paranoid ideas, tendency of the affect to remain warm and appropriate, and no typical deterioration to the hebephrenic state [27]. Patients had no history of previous psychiatric disorders (prior to seizure onset) or of substance dependence at any time. 
Global IQ was calculated after neuropsychological tests (complete Wechsler Adult Intelligence Scale, version III (WAIS-III) or WAIS-R protocol).

\section{Tissue collection and immunohistochemical processing}

Specimens were segmented into 1 -cm blocks transversely oriented to the hippocampal long axis. Blocks were placed in buffered paraformaldehyde (Sigma, St Louis, MO, USA). After 48 to $96 \mathrm{~h}$, specimens were paraffin embedded for immunohistochemistry.

Immunohistochemistry was performed with antibodies that identified immunoreactivity for reactive astrocytes (GFAP, 1:500 dilution; Dako, Glostrup, Denmark), activated microglia (human leukocyte antigen, MHC class II (HLA-DR), 1:100 dilution; Dako, Glostrup, Denmark), astroglial metallothionein I/II (MT-I/II, 1:500 dilution, Dako, Glostrup, Denmark), and perivascular aquaporin 4 (AQP4, 1:200 dilution; Santa Cruz Biotechnology, Santa Cruz, CA, USA). Antibodies specificity was verified, and immunohistochemistry was performed as described in Peixoto-Santos et al. [4]. Briefly, paraffin-embedded MTLE and control hippocampi were processed together for each antibody, with overnight incubation at room temperature, and developed simultaneously in $0.05 \% 3,3^{\prime}$ diaminobenzidine tetrahydrochloride (Pierce, Rockford, USA) and $0.01 \%$ hydrogen peroxide (Merck, Darmstadt, Germany). After sufficient colorization, reaction was halted by washing in several rinses of distilled water, dehydrated through graded ethanol to xylene (Merck, Darmstadt, Germany), and cover slipped with Krystalon (EM Science, Gibbstown, NJ, USA). Adjacent sections were hematoxilineosin stained (Laborclin, Pinhais, Brazil) and examined for tissue integrity. Control sections without the primary antisera did not reveal staining (data not shown).

\section{Semi-quantitative analysis of immunohistochemistry}

MTLE and control hippocampi were compared for immunoreactivity in several hippocampal formation subfields using Lorente de No's classification [28], which included fascia dentata granular cells and hilar neurons, as well as pyramidal cells in the cornus ammonis region 4 (CA4), cornus ammonis region 3 (CA3), cornus ammonis region 2 (CA2), cornus ammonis region 1 (CA1), prosubiculum, subiculum, parasubiculum, and entorhinal cortex layer III. Immunoreactivity were estimated in $8-\mu \mathrm{m}$ Neu-N stained slices at $\times 200$ magnification as previously described and well established in the literature for surgical hippocampal fragments $[1,3,4,29,30]$.

Images of each hippocampal formation subfield from all specimens were collected and digitized with a highresolution $\mathrm{CCD}$ monochrome camera attached to an Olympus microscope. Uniform luminance was maintained and checked every ten measurements using an optical density standard and a gray value scale ranging from 0 (white) to 255 (black). In brief, all digitized images were analyzed with Image J software, following the same criteria: (I) the software identifies the gray value distribution of a subfield's digital image; (II) the immunoreactive area is selected (that is, positive stained pixels), limited to a threshold range; and (III) the threshold range is pre-settled based on control group sections, to exclude the low-intensity gray value of background staining from the analysis. For GFAP, the threshold selected allowed the quantification of positive staining present in the soma, branches, and also of the fine and characteristic astroglial meshwork. For HLA-DR, the threshold selected allowed the quantification of proteins present in the soma and branches of the immunostained cells, whereas the MT-I/II threshold allowed the quantifications of proteins in the soma and proximal branches of astrocytes. As for AQP4, we selected a higher threshold, in order to quantify only AQP4 present in the endfeets of astrocytes (that is, perivascular AQP4). A similar approach was used by our group elsewhere [3,4]. Analyses were conducted blind to hippocampal pathology and group classification.

\section{Data analysis}

Data were analyzed using the statistical program PASW (version 18.0) and SigmaPlot (version 11.0). Groups were compared using analysis of variance (ANOVA one way, with Bonferroni post hoc test) or unpaired $t$ test for variables with normal distribution and Kruskal-Wallis One Way Analysis of Variance on Ranks (with Dunn post hoc test) or Mann-Whitney Rank Sum Test for variables without normal distribution. The Fisher Exact test was applied for comparison of relative frequencies of clinical variables between groups. Statistical significance was set at $P<0.05$ and values presented as mean \pm standard deviation (SD).

\section{Results}

\section{Clinical profiles}

The four patient groups did not show significant differences in gender, age, or collected side (Table 1). Clinical variables such as presence of an initial precipitant injury, age of first seizure and seizure onset, seizure frequency, type and outcome, epilepsy duration, HS side, handedness, IQ, years at school, and performance in neuropsychological tests were homogeneously distributed among MTLE groups.

All epileptic patients were on antiepileptic drugs (carbamazepine, oxcarbazepine, phenobarbital, and/or phenytoin). In addition, patients were also taking benzodiazepines $\left(\right.$ MTLE $_{W}$ group: 11 of 17; MTLE $+\mathrm{D}$ group: 10 of 15; MTLE + P group: 8 of 11), fluoxetine (MTLE + D group: 5 of 15), and haloperidol (MTLE + P group: 6 of 11). No differences in neuropsychological tests 
between patients taking or not taking benzodiazepines, fluoxetine, or haloperidol were seen. No significant influence of fluoxetine or haloperidol was seen on hippocampal GFAP, HLA-DR, or AQP4 expression. Haloperidol influence on MT-I/II expression will be described below.

\section{Reactive astrocytes}

Immunohistochemistry for GFAP, a marker of reactive astrocytes, showed a higher number of immunopositive cells and astrocytic processes in MTLE patients (Figure 1A, $\mathrm{B}, \mathrm{C})$, compared to staining in the controls (Figure 1D). Evaluation of the immunopositive area fraction (Figure 2A) revealed a higher GFAP area in all MTLE groups in the outer molecular layer, inner molecular layer, granule cell layer, hilus, CA4, CA3, CA1, prosubiculum, subiculum, and parasubiculum $(P \leq 0.024)$ when compared to controls.
$\mathrm{MTLE}_{\mathrm{W}}$ and MTLE + P had also increased immunopositive area in the inner molecular layer, granule cell layer, and CA2 $(P \leq 0.049)$ compared to MTLE + D. In CA1, MTLE had a higher immunopositive area than MTLE + D $(P<$ $0.001)$. In the outer molecular layer, MTLE $+P$ had a higher GFAP immunopositive area than MTLE + D $(P=0.013)$. In the entorhinal cortex, only the groups with psychiatric comorbidities (that is, MTLE $+\mathrm{D}$ and MTLE $+\mathrm{P}$ ) had increased GFAP immunopositive area when compared to controls $(P \leq 0.032)$.

\section{Activated microglia}

Activated microglia, evaluated with antibody against HLA-DR, was observed in MTLE patients as small, highly branched cells, well defined and spaced from each other (Figure 1E,F,G). In control specimens, activated
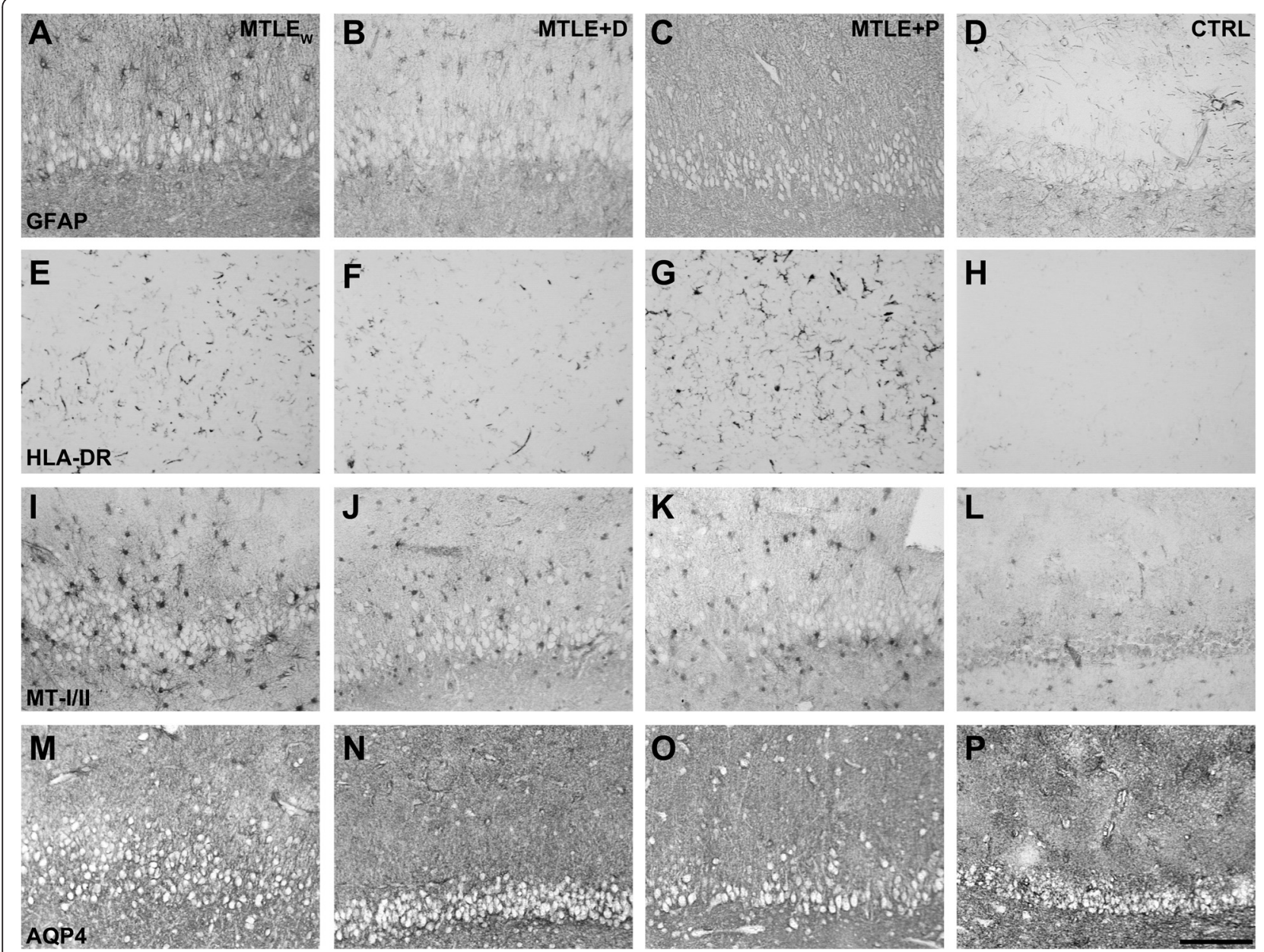

Figure 1 Representative images of fascia dentata immunostained for GFAP (A, B, C, D), HLA-DR (E, F, G, H), MT-I/II (I, J, K, L), and AQP4 $(M, N, O, P)$ from patients with $\operatorname{MTLE}_{W}(A, E, I, M), M T L E+D(B, F, J, N), M T L E+P(C, G, K, O)$ and autopsy controls (D, H, L, P). Observe the increased astroglial reaction $(A, B, C)$, microglial activation $(E, F, G), M T-I / I I$ immunopositive astrocytes $(I, J, K)$, and reduced perivascular aquaporin 4 $(M, N, O)$ in MTLE groups (MTLE,$M T L E+D$, and MTLE + P), when compared to the respective staining pattern of the $C T R L$ group $(D, H, L$, $P)$. Bar in (P) indicates $150 \mu \mathrm{m}$. AQP4 = aquaporin 4; CTRL = control; GFAP = glial fibrillary acidic protein; HLA-DR= human leukocyte antigen, MHC class II; MT-I/II = metallothionein-I/I; MTLE = mesial temporal lobe epilepsy; MTLE + D = MTLE + major depression; MTLE + P = MTLE + interictal psychosis; MTLE $=$ MTLE subjects without psychiatric history. 


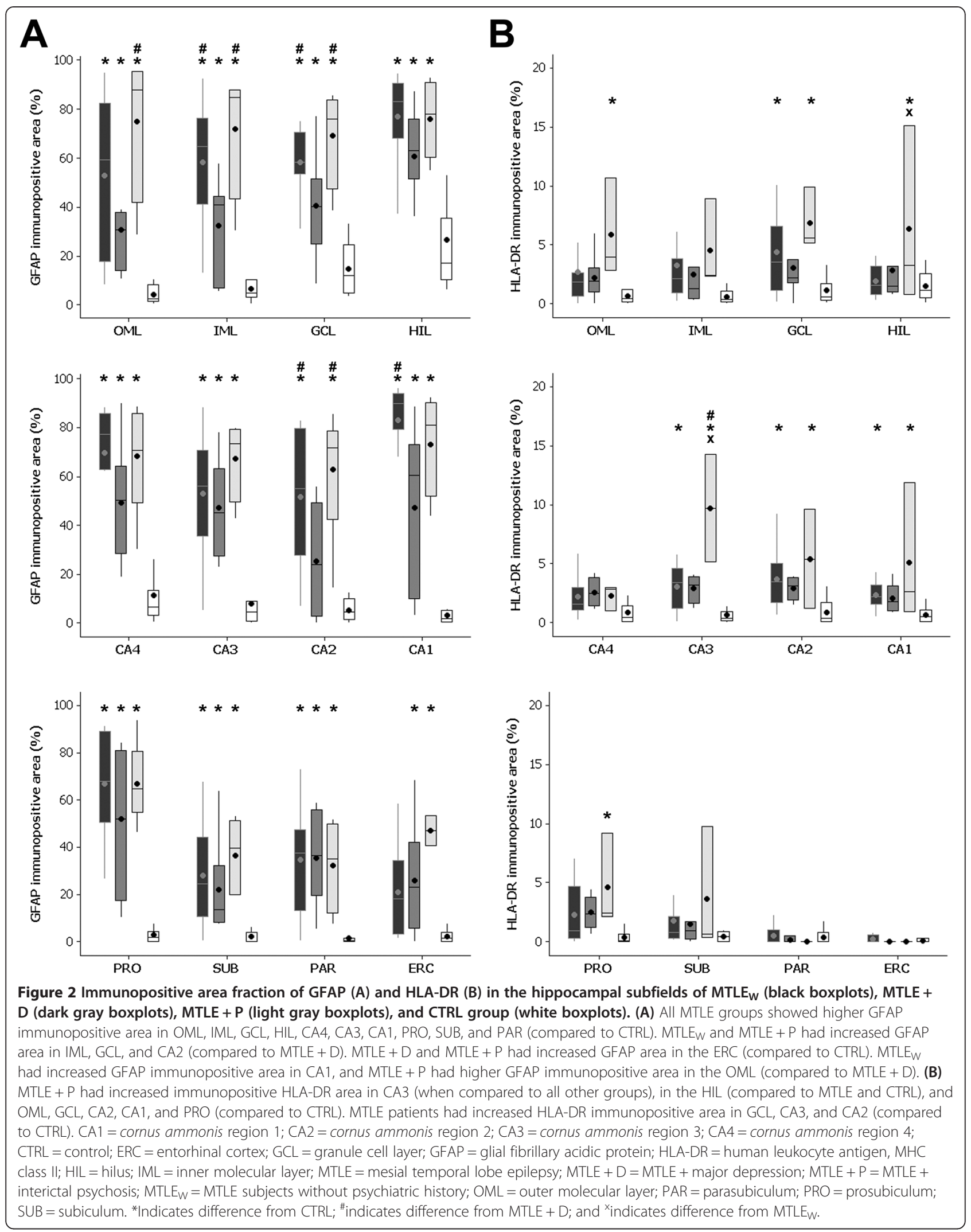


microglia were rarely seen and, when present, were in much smaller number than in MTLE cases (Figure $1 \mathrm{H}$ ). Quantitative evaluation of activated microglia (Figure 2B) revealed increased immunopositivity in the outer molecular layer, granule cell layer, hilus, CA3, CA2, CA1, and prosubiculum of MTLE $+\mathrm{P}$ when compared to the control $(P \leq 0.047)$. Patients of the MTLE $+P$ group had also a higher immunopositive area in the hilus when compared to $\operatorname{MTLE}_{\mathrm{W}}(P=0.04)$, and in CA3 when compared to MTLE $\mathrm{W}_{\mathrm{W}}$ and MTLE + D $(P \leq 0.002)$. MTLE patients had an increased HLA-DR immunopositive area in the granule cell layer, CA3, CA2, and CA1 when compared to the control $(P \leq 0.038)$.

\section{Metallothioneins I/II}

The immunopositive staining for MT-I/II was observed in cells with astroglial morphology (see Figure 1I,J,K,L). Although only patients from the MTLE $\mathrm{W}_{\mathrm{W}}$ group had significant increase in MT-I/II immunopositive cells, qualitatively, all MTLE groups present a higher number of MT-I/II positivity in comparison to the control group (compare the micrography $\mathrm{L}$ with the micrographies I to $\mathrm{K}$ in Figure 1). Higher immunopositive staining (Figure 3A) was observed in the inner molecular layer, CA2, CA1, parasubiculum, and entorhinal cortex of $\mathrm{MTLE}_{\mathrm{W}}$ when compared to the control group $(P \leq 0.015)$. Compared to MTLE + P, higher immunopositive area was observed in the granule cell layer, the CA1, and the parasubiculum of MTLE $_{\mathrm{W}}$ cases $(P \leq 0.019)$. In the parasubiculum, the group MTLE $_{W}$ had a higher area fraction than the group MTLE + D $(P<0.001)$.

MT-I/II expression was increased in the inner molecular layer of the MTLE $+\mathrm{P}$ patients taking haloperidol when compared to those not taking it (with haloperidol, mean area fraction $=7.5 \pm 3.5$; without haloperidol, mean area fraction $=1.7 \pm 2.2 ; t(9)=2.885 ; P=0.02$ ). Also, we found a trend of increased CA2 MT-I/II expression in patients who achieved complete seizure remission after surgery (remission, mean area fraction $=20.3 \pm 24.6$; noremission, mean area fraction $=9.1 \pm 6.2 ; t(38)=-1.946$; $P=0.06)$.

\section{Aquaporin 4}

AQP4 immunohistochemistry revealed a reduction in the perivascular staining intensity in MTLE specimens, when compared to controls (compare micrography $\mathrm{P}$ with micrographies $\mathrm{M}$ to $\mathrm{O}$ in Figure 1). Compared to the control, all MTLE groups had decreased perivascular AQP4 area fraction in the hilus $(P \leq 0.015)$, whereas in CA4, only MTLE $+\mathrm{P}$ showed significant decrease $(P=$ 0.026), and in the subiculum, MTLE $\mathrm{W}_{\mathrm{W}}$ and MTLE $+\mathrm{P}$ had reduced immunopositive area $(P \leq 0.015)$. A direct correlation was seen between IQ and AQP4 expression in the CA1 $(R=0.530 ; P=0.006)$ and in the prosubiculum
$(R=0.529 ; P=0.008)$ of MTLE patients. Also, we found a trend to increased AQP4 expression in the CA2 of those patients who achieved complete seizure remission after surgery (remission, mean area fraction $=9.9 \pm 9.0$; noremission, mean area fraction $=5.3 \pm 2.9 ; t(38)=-1.930$; $P=0.07)$.

\section{Discussion}

In the present study, we investigated the expression of glial proteins GFAP, HLA-DR, MT-I/II, and perivascular AQP4 in the hippocampal formation of MTLE with and without psychiatric comorbidities and in non-epileptic controls. Comparing the MTLE groups, we found in specific hippocampal subfields an increased immunoreactive area of GFAP and HLA-DR and decreased MT-I/ II and AQP4 in specimens from the MTLE patients with psychosis; while in specimens from patients with MTLE and major depression, GFAP and MT-I/II were decreased. Differences between MTLE and controls, in astrogliosis, microgliosis, increased MT-I/II, and decreased perivascular AQP4 in the epileptogenic hippocampus, were similar to what is currently described in the literature $[4,31]$. Given that differences between epileptogenic and control hippocampi are already well established in the literature, our discussion will focus mainly on psychiatric subgroups and their differences when compared to MTLE without psychiatric comorbidities, unless otherwise specified.

Studies in humans and animal models of epilepsy have shown upregulation of several inflammatory molecules $[32,33]$. However, only a few experimental studies have focused on inflammatory changes in correlates of major depression comorbid with epilepsy. For example, rats injected with pilocarpine exhibit behavioral equivalents of anhedonia and despair and alterations in inflammatory molecules as found in human major depression $[34,35]$. No information regarding neuroinflammatory mechanisms in psychosis of epilepsy is available to date.

Astrogliosis and microgliosis are part of a common response to injury. Although the reactive astrocyte expression profile may depend on the type of inducing injury, increased inflammatory-related molecules are always found in reactive astrocytes [36]. Interleukins $1 \mathrm{~b}$ (IL1beta) and 6 (IL-6) are among the molecules released after injury that can lead to glial reaction [37]. In fact, the crosstalk between activated microglia and reactive astrocytes seems crucial to the maintenance of chronic gliosis [38]. Increased astrocytic GFAP expression, a marker of reactive astrogliosis, is a common finding in the hippocampus of MTLE patients [3,4]. Likewise, we detected an increased GFAP immunoreactive area in all hippocampal subfields of MTLE patients. In patients with MTLE + D, GFAP expression levels were intermediary between the controls and $\mathrm{MTLE}_{\mathrm{W}}$ or MTLE + P. 


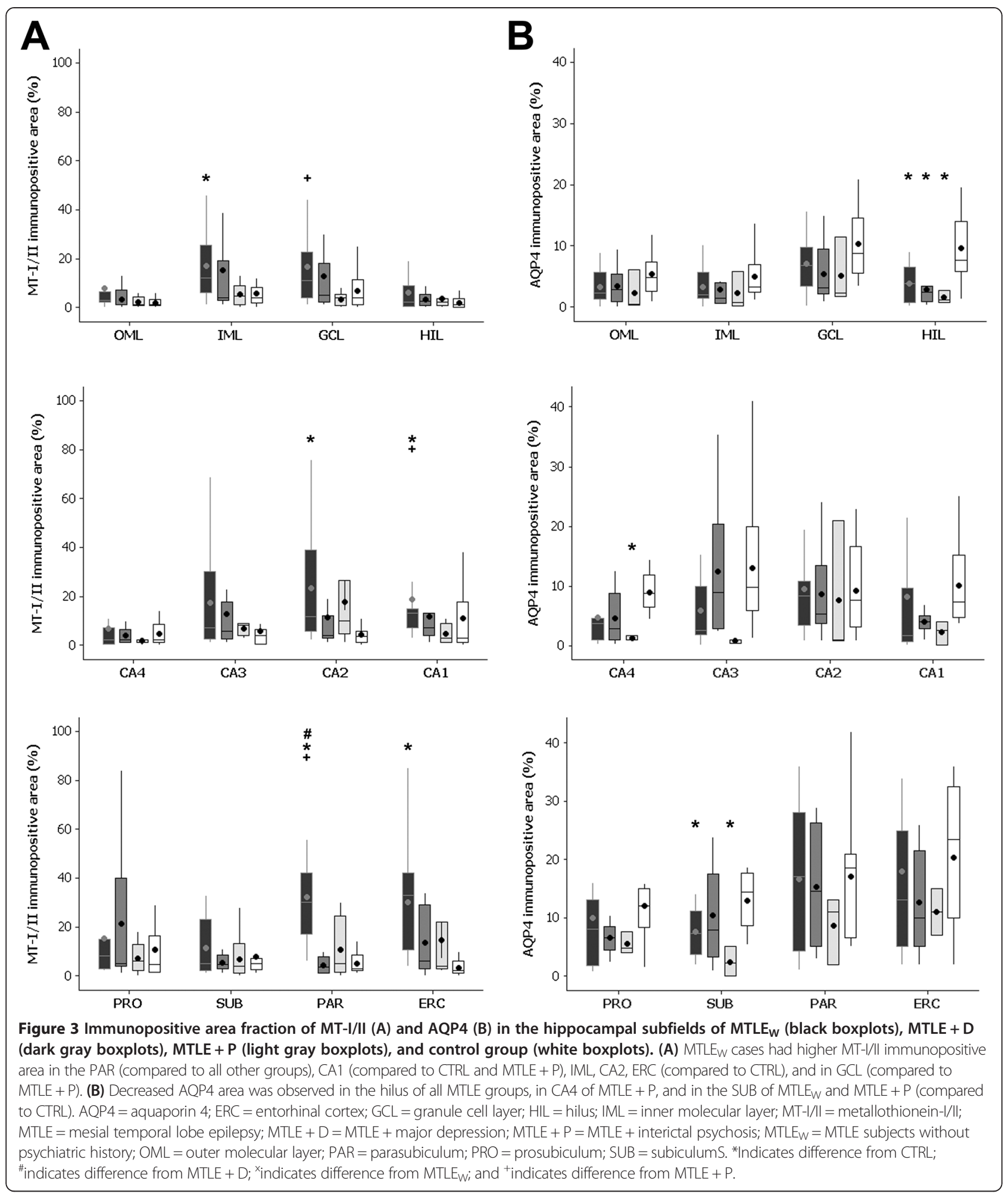

Since studies have shown that patients with major depression have reduced GFAP expression [18,39], it is possible that the mechanisms underlying the decreased GFAP expression observed in major depression counterbalance the increase found in epilepsy, resulting in the intermediary values observed in our MTLE + D cases. In schizophrenia, a study showed that increased GFAP expression in the prefrontal cortex of patients with schizophrenia is increased [16], but other cortical areas and the hippocampus have shown inconclusive 
results $[17,40,41]$. In MTLE patients with interictal psychosis, we found increased GFAP expression, especially when compared to the MTLE + D cases, in agreement with a recent hypothesis that astrocyte pathology may be associated with psychotic symptoms, although the exact nature of this change remains unclear [16]. In particular, increased GFAP in schizophrenia/psychotic symptoms could be closely related to increased neuroinflammatory markers [42], as well as to increased IL-1beta and IL-6 serum levels [43]. A recent study comparing MTLE hippocampi from patients with and without de novo psychosis (postoperative psychosis) analyzed GFAP expression and found no qualitative differences between groups [44]. In our present series, quantitative differences in GFAP between $M_{T L E}$ and MTLE + P were also subtle, and major differences were seen in respect to the MTLE $+\mathrm{D}$ group.

Increased cortical and hippocampal HLA-DR+ microglia has been described in schizophrenia $[17,45]$, in accordance to our findings in several hippocampal subfields of MTLE patients with interictal psychosis. Of note, increased hippocampal HLA-DR was particularly associated with paranoid schizophrenia [45], a core symptom especially represented in interictal psychosis [20]. HLA-DR levels in MTLE specimens from patients without psychiatric comorbidities and in those with major depression were similar and higher than in the controls, although statistically significant differences were detected only in the granule cell layer and CA3-1 of MTLE $\mathrm{W}_{\mathrm{W}}$ versus control. Microglia is an important source of inflammatory molecules [32], and a high expression of pro-inflammatory cytokines is observed in major depression [46]. Similar apparent microglial activation in MTLE $\mathrm{W}_{\mathrm{W}}$ and MTLE + D could partially explain why patients with epilepsy frequently develop mood disorders, an association still incompletely understood [47]. However, the levels of microglial-related inflammatory molecules such as cytokines remain to be evaluated in human epilepsy with and without major depression.

Metallothioneins are regulators of free zinc levels, an important modulator of glutamatergic neurotransmission [12]. Besides metals, oxidative stress agents and inflammatory molecules can induce MT-I/II expression [12]. Knockout mice for IL-6 have low microglial activation and low expression of MT-I/II, indicating a crucial role of inflammation in MT-I/II expression [48]. In fact, mice overexpressing MT-I/II show reduced microgliosis and reduced levels of interleukins following kainic acid status epilepticus [49]. In psychiatric diseases, MT-I/II gene expression in the prefrontal cortex has been found increased in schizophrenia [15] and decreased in major depression [19]. No reports are available regarding the hippocampus, but in our series, we found decreased values in cases with psychosis and in those with major depression when compared to MTLE without psychiatric comorbidities in several hippocampal subfields. Interestingly, we have found in other series of patients decreased mossy fiber sprouting in MTLE $+\mathrm{P}$ and increased in MTLE + D $[8,9]$. Since mossy fibers are zinc enriched and MT-I/II chelates zinc, cadmium, and copper, it would be expected that hippocampi from MTLE $+\mathrm{D}$ have a deficient metal homeostasis and likely zinc excess in neurons and glial cells and in the neuropile. A possible mechanism would be through zinc overflow from serum to brain [50] due to an inefficient blood-brain barrier in major depression [51]. In fact, low-serum zinc is a hallmark of major depressive disorders [52]. Our results of decreased hippocampal MT-I/II in MTLE associated to psychosis can be related to decreased hippocampal zinc levels/mossy fibers in interictal psychosis [8,9], as well as in schizophrenia [53,54]. Interestingly, MTLE + P patients taking haloperidol showed increased expression of MT-I/ II in the inner molecular layer. In the amphetamine animal model of schizophrenia, zinc administration is able to revert behavioral equivalents of positive symptoms [55], but it is unknown if systemic zinc administration alters hippocampal zinc and/or MT-I/II expression. In fact, zinc is a ligand of the haloperidol-sensitive sigma 2 receptor in the mossy fiber of rats [56], suggesting that an increased MT-I/II expression in the mossy fiber of MTLE + P patients would facilitate zinc chelation and proper haloperidol binding. We also found a trend to increased MT-I/II in the CA2 of patients who achieved complete seizure remission after surgery, in agreement to the MT-I/II role in the control of excitability [57]. Likewise, other recent evidences indicate that hippocampal expression of proteins used as markers of full-blown epileptogenesis might be able to predict seizure outcome [58,59].

AQP4 is the main water channel in the central nervous system and presents with multifaceted functions. In inflammatory conditions, microglia can release IL-1beta, which in turn induces AQP4 expression in astrocytes [60]. AQP4 is able to regulate brain response to insults or injury, and also to influence synaptic plasticity and behavior [61]. In the AQP4 knockout mouse, memory is impaired [62]. In accordance, our results showed a direct correlation between perivascular AQP4 expression in the Sommer sector and IQ scores. AQP4 participation in synaptic plasticity and cognition occurs together with neurotrophin (NT) receptors [62]. Of note, NTs and NT receptors are differentially regulated in MTLE with psychiatric comorbidities $[9,58]$, which could further change how AQP4 modulates plasticity. For instance, the brainderived neurotrophic factor (BDNF) is increased in MTLE $_{W}$ but decreased in MTLE + P [9]. In addition, tyrosine kinase receptor type 2 (TrkB) (a BDNF receptor) is increased in MTLE + P but not in MTLE $\mathrm{W}_{\mathrm{W}}$ [58]. Given that low levels of AQP4 associated with increased BDNF and TrkB or p75 neurotrophin receptor (p75NTR) may 
result in increased excitability, AQP4 levels near to control levels could be more efficient in controlling excessive excitatory activation trough a BDNF-TrkB or p75NTR loop $[62,63]$. In fact, we found a trend to increased AQP4 in cases with complete seizure remission, thus reinforcing the role of AQP4 in neuron activity. In addition, AQP4 has an important role in $\mathrm{K}^{+}$homeostasis [13,64], and AQP4 knockout mice have higher seizure threshold but longer seizure duration [13].

\section{Conclusion}

In summary, we described hippocampal neuroinflammatoryrelated molecules that show a distinct pattern of expression when MTLE patients present with a comorbid psychiatric diagnosis of interictal psychosis or major depression. Studies have reported successful treatment of patients with seizures, schizophrenia, and major depression using drugs with anti-inflammatory effect as an add-on therapy [65-68]. Given the differential expression of neuroinflammatoryrelated molecules in MTLE with psychiatric comorbidities, these patients could also benefit from a more targeted treatment. Further research is needed to expand and validate these findings and to better investigate possible causal mechanisms.

\begin{abstract}
Abbreviations
AQP4: aquaporin 4; BDNF: brain-derived neurotrophic factor; CA1: cornus ammonis region 1; CA2: cornus ammonis region 2; CA3: cornus ammonis region 3; CA4: cornus ammonis region 4; EEG: electroencephalogram; GFAP: glial fibrillary acidic protein; HLA-DR: human leukocyte antigen, MHC class II; HS: hippocampal sclerosis; IQ: intelligence quotient; MT-I/ II: metallothionein I and II; MTLE: mesial temporal lobe epilepsy; MTLE + D: MTLE + major depression; MTLE + P: MTLE + interictal psychosis; NT: neurotrophin; p75NTR: p75 neurotrophin receptor; SD: standard deviation; TrkB: tyrosine kinase receptor, type 2; WAIS-III: Wechsler Adult Intelligence Scale, version III.
\end{abstract}

\section{Competing interests}

The authors declare that they have no competing interests.

\section{Authors' contributions}

Conception and design of research (LK); performed research (LK, JEPS, MRM, RCS); analyzed data (JEPS); contributed with reagents/analytic tools and/or important intellectual input (JAA, CGC, JEH); wrote the manuscript (LK, JEPS, JPL). All authors read and approved the final manuscript.

\section{Acknowledgements}

This work was supported by Fundacao de Apoio a Pesquisa do Estado de Sao Paulo - Fapesp (CInAPCe Project 05/56447-7, to JPL; PhD fellowship 2010/ 51515-2, to JEPS; PhD fellowship 2011/23691-3, to MRM), Conselho Nacional de Desenvolvimento Cientifico e Tecnologico - CNPq, and Coordenacao de Aperfeicoamento de Pessoal de Nivel Superior - CAPES (postdoc fellowship A034-2013, to LK).

\section{Disclosures}

The funders had no role in study design, data collection and analysis, decision to publish, or preparation of the manuscript.

\section{Author details}

${ }^{1}$ Department of Neurosciences and Behavior, Ribeirao Preto Medical School, University of Sao Paulo (USP), Av Bandeirantes 3900, CEP 14049-900 Ribeirao Preto, SP, Brazil. ${ }^{2}$ Center for Interdisciplinary Research on Applied Neurosciences (NAPNA), USP, Ribeirao Preto, Brazil. ${ }^{3}$ Department of Surgery, Ribeirao Preto Medical School, USP, Ribeirao Preto, Brazil. ${ }^{4}$ National Institute of Science and Technology in Translational Medicine (INCT-TM - CNPq), Ribeirao Preto, Brazil.

Received: 4 October 2014 Accepted: 10 February 2015

Published online: 25 February 2015

\section{References}

1. Mathern GW, Babb TL, Pretorius JK, Leite JP. Reactive synaptogenesis and neuron densities for neuropeptide $Y$, somatostatin, and glutamate decarboxylase immunoreactivity in the epileptogenic human fascia dentata. J Neurosci. 1995;15:3990-4004.

2. Babb TL, Kupfer WR, Pretorius JK, Crandall PH, Levesque MF. Synaptic reorganization by mossy fibers in human epileptic fascia dentata. Neuroscience. 1991;42:351-63.

3. Kandratavicius L, Rosa-Neto P, Monteiro MR, Guiot MC, Assirati Jr JA, Carlotti Jr CG, et al. Distinct increased metabotropic glutamate receptor type 5 (mGluR5) in temporal lobe epilepsy with and without hippocampal sclerosis. Hippocampus. 2013;23:1212-30.

4. Peixoto-Santos JE, Galvis-Alonso OY, Velasco TR, Kandratavicius L, Assirati JA, Carlotti CG, et al. Increased metallothionein I/I expression in patients with temporal lobe epilepsy. PLoS One. 2012;7:e44709.

5. Qin P, Xu H, Laursen TM, Vestergaard M, Mortensen PB. Risk for schizophrenia and schizophrenia-like psychosis among patients with epilepsy: population based cohort study. BMJ. 2005;331:23.

6. Kandratavicius L, Lopes-Aguiar C, Bueno-Junior LS, Romcy-Pereira RN, Hallak $J E$, Leite JP. Psychiatric comorbidities in temporal lobe epilepsy: possible relationships between psychotic disorders and involvement of limbic circuits. Rev Bras Psiquiatr. 2012;34:454-66.

7. Kandratavicius L, Ruggiero RN, Hallak JE, Garcia-Cairasco N, Leite JP. Pathophysiology of mood disorders in temporal lobe epilepsy. Rev Bras Psiquiatr. 2012;34 Suppl 2:S233-45.

8. Kandratavicius L, Hallak JE, Young LT, Assirati JA, Carlotti Jr CG, Leite JP. Differential aberrant sprouting in temporal lobe epilepsy with psychiatric co-morbidities. Psychiatry Res. 2012;195:144-50.

9. Kandratavicius L, Monteiro MR, Assirati Jr JA, Carlotti Jr CG, Hallak JE, Leite JP. Neurotrophins in mesial temporal lobe epilepsy with and without psychiatric comorbidities. J Neuropathol Exp Neurol. 2013;72:1029-42.

10. Kandratavicius L, Monteiro MR, Hallak JE, Carlotti Jr CG, Assirati Jr JA, Leite JP. Microtubule-associated proteins in mesial temporal lobe epilepsy with and without psychiatric comorbidities and their relation with granular cell layer dispersion. Biomed Res Int. 2013;2013:960126.

11. Najjar S, Pearlman DM, Alper K, Najjar A, Devinsky O. Neuroinflammation and psychiatric illness. J Neuroinflammation. 2013;10:43.

12. Ebadi M, Iversen PL, Hao R, Cerutis DR, Rojas P, Happe HK, et al. Expression and regulation of brain metallothionein. Neurochem Int. 1995;27:1-22.

13. Binder DK, Yao X, Zador Z, Sick TJ, Verkman AS, Manley GT. Increased seizure duration and slowed potassium kinetics in mice lacking aquaporin-4 water channels. Glia. 2006;53:631-6.

14. Jukkola P, Guerrero T, Gray V, Gu C. Astrocytes differentially respond to inflammatory autoimmune insults and imbalances of neural activity. Acta Neuropathol Commun. 2013;1:70-89.

15. Choi KH, Elashoff M, Higgs BW, Song J, Kim S, Sabunciyan S, et al. Putative psychosis genes in the prefrontal cortex: combined analysis of gene expression microarrays. BMC Psychiatry. 2008;8:87.

16. Feresten AH, Barakauskas V, Ypsilanti A, Barr AM, Beasley CL. Increased expression of glial fibrillary acidic protein in prefrontal cortex in psychotic illness. Schizophr Res. 2013;150:252-7.

17. Radewicz K, Garey L, Gentleman SM, Reynolds R. Increase in HLA-DR immunoreactive microglia in frontal and temporal cortex of chronic schizophrenics. J Neuropathol Exp Neurol. 2000;59:137-50.

18. Rajkowska G, Stockmeier CA. Astrocyte pathology in major depressive disorder: insights from human postmortem brain tissue. Curr Drug Targets. 2013;14:1225-36.

19. Shelton RC, Claiborne J, Sidoryk-Wegrzynowicz M, Reddy R, Aschner M, Lewis DA, et al. Altered expression of genes involved in inflammation and apoptosis in frontal cortex in major depression. Mol Psychiatry. 2011;16:751-62.

20. Kandratavicius L, Hallak JE, Leite JP. What are the similarities and differences between schizophrenia and schizophrenia-like psychosis of epilepsy? A neuropathological approach to the understanding of schizophrenia spectrum and epilepsy. Epilepsy Behav. 2014;38C:143-7. 
21. Gittins R, Harrison PJ. Neuronal density, size and shape in the human anterior cingulate cortex: a comparison of Nissl and NeuN staining. Brain Res Bull. 2004;63:155-60.

22. Stan AD, Ghose S, Gao XM, Roberts RC, Lewis-Amezcua K, Hatanpaa KJ, et al. Human postmortem tissue: what quality markers matter? Brain Res. 2006;1123:1-11.

23. Berg AT. Identification of pharmacoresistant epilepsy. Neurol Clin. 2009:27:1003-13.

24. Engel Jr J. Surgery for seizures. N Engl J Med. 1996;334:647-52.

25. Sachdev P. Schizophrenia-like psychosis and epilepsy: the status of the association. Am J Psychiatry. 1998;155:325-36.

26. Elliott B, Joyce E, Shorvon S. Delusions, illusions and hallucinations in epilepsy: 2. Complex phenomena and psychosis. Epilepsy Res. 2009:85:172-86.

27. Beard AW, Slater E. The schizophrenic-like psychoses of epilepsy. Proc R Soc Med. 1962;55:311-6.

28. LorentedeNo R. Studies on the structure of the cerebral cortex II: continuation of study of the ammonic system. J Psychol Neurol. 1934:46:113-77.

29. Mathern GW, Mendoza D, Lozada A, Pretorius JK, Dehnes Y, Danbolt NC, et al. Hippocampal GABA and glutamate transporter immunoreactivity in patients with temporal lobe epilepsy. Neurology. 1999;52:453-72.

30. Mathern GW, Pretorius JK, Kornblum HI, Mendoza D, Lozada A, Leite JP, et al. Human hippocampal AMPA and NMDA mRNA levels in temporal lobe epilepsy patients. Brain. 1997;120(Pt 11):1937-59.

31. Eid T, Lee TS, Thomas MJ, Amiry-Moghaddam M, Bjornsen LP, Spencer DD, et al. Loss of perivascular aquaporin 4 may underlie deficient water and $\mathrm{K}+$ homeostasis in the human epileptogenic hippocampus. Proc Natl Acad Sci U S A. 2005;102:1193-8.

32. Arisi GM. Nervous and immune systems signals and connections: cytokines in hippocampus physiology and pathology. Epilepsy Behav. 2014;38C:43-7.

33. Vezzani A, Ravizza T, Balosso S, Aronica E. Glia as a source of cytokines: implications for neuronal excitability and survival. Epilepsia. 2008:49 Suppl 2:24-32.

34. Pineda E, Shin D, Sankar R, Mazarati AM. Comorbidity between epilepsy and depression: experimental evidence for the involvement of serotonergic, glucocorticoid, and neuroinflammatory mechanisms. Epilepsia. 2010;51 Suppl 3:110-4.

35. Xie W, Cai L, Yu Y, Gao L, Xiao L, He Q, et al. Activation of brain indoleamine 2,3-dioxygenase contributes to epilepsy-associated depressive-like behavior in rats with chronic temporal lobe epilepsy. J Neuroinflammation. 2014;11:41.

36. Zamanian JL, Xu L, Foo LC, Nouri N, Zhou L, Giffard RG, et al. Genomic analysis of reactive astrogliosis. J Neurosci. 2012;32:6391-410.

37. Hostenbach S, Cambron M, D'Haeseleer M, Kooijman R, De Keyser J. Astrocyte loss and astrogliosis in neuroinflammatory disorders. Neurosci Lett. 2014:565:39-41.

38. Liu W, Tang Y, Feng J. Cross talk between activation of microglia and astrocytes in pathological conditions in the central nervous system. Life Sci. 2011;89:141-6.

39. Muller MB, Lucassen PJ, Yassouridis A, Hoogendijk WJ, Holsboer F, Swaab DF. Neither major depression nor glucocorticoid treatment affects the cellular integrity of the human hippocampus. Eur J Neurosci. 2001;14:1603-12.

40. Steffek AE, McCullumsmith RE, Haroutunian V, Meador-Woodruff JH. Cortical expression of glial fibrillary acidic protein and glutamine synthetase is decreased in schizophrenia. Schizophr Res. 2008;103:71-82.

41. Webster MJ, Knable MB, Johnston-Wilson N, Nagata K, Inagaki M, Yolken RH. Immunohistochemical localization of phosphorylated glial fibrillary acidic protein in the prefrontal cortex and hippocampus from patients with schizophrenia, bipolar disorder, and depression. Brain Behav Immun. 2001;15:388-400.

42. Catts VS, Wong J, Fillman SG, Fung SJ, Weickert CS. Increased expression of astrocyte markers in schizophrenia: association with neuroinflammation. Aust N Z J Psychiatry. 2014:48:722-34.

43. Song X, Fan X, Song X, Zhang J, Zhang W, Li X, et al. Elevated levels of adiponectin and other cytokines in drug naive, first episode schizophrenia patients with normal weight. Schizophr Res. 2013;150:269-73.

44. Thom M, Kensche M, Maynard J, Liu J, Reeves C, Goc J, et al. Interictal psychosis following temporal lobe surgery: dentate gyrus pathology. Psychol Med. 2014;44:3037-49.
45. Busse S, Busse M, Schiltz K, Bielau H, Gos T, Brisch R, et al. Different distribution patterns of lymphocytes and microglia in the hippocampus of patients with residual versus paranoid schizophrenia: further evidence for disease course-related immune alterations? Brain Behav Immun 2012;26:1273-9.

46. Rosenblat JD, Cha DS, Mansur RB, McIntyre RS. Inflamed moods: a review of the interactions between inflammation and mood disorders. Prog Neuropsychopharmacol Biol Psychiatry. 2014;53C:23-34

47. Kanner AM. Can neurobiological pathogenic mechanisms of depression facilitate the development of seizure disorders? Lancet Neurol. 2012;11:1093-102.

48. Penkowa M, Molinero A, Carrasco J, Hidalgo J. Interleukin-6 deficiency reduces the brain inflammatory response and increases oxidative stress and neurodegeneration after kainic acid-induced seizures. Neuroscience. 2001;102:805-18

49. Penkowa M, Florit S, Giralt M, Quintana A, Molinero A, Carrasco J, et al. Metallothionein reduces central nervous system inflammation, neurodegeneration, and cell death following kainic acid-induced epileptic seizures. J Neurosci Res. 2005;79:522-34.

50. Takeda A. Movement of zinc and its functional significance in the brain. Brain Res Brain Res Rev. 2000;34:137-48.

51. Najjar S, Pearlman DM, Devinsky O, Najjar A, Zagzag D. Neurovascular unit dysfunction with blood-brain barrier hyperpermeability contributes to major depressive disorder: a review of clinical and experimental evidence. J Neuroinflammation. 2013;10:142.

52. Szewczyk B, Kubera M, Nowak G. The role of zinc in neurodegenerative inflammatory pathways in depression. Prog Neuropsychopharmacol Biol Psychiatry. 2011;35:693-701.

53. Goldsmith SK, Joyce JN. Alterations in hippocampal mossy fiber pathway in schizophrenia and Alzheimer's disease. Biol Psychiatry. 1995;37:122-6.

54. Kolomeets NS, Orlovskaya DD, Uranova NA. Decreased numerical density of CA3 hippocampal mossy fiber synapses in schizophrenia. Synapse. 2007;61:615-21.

55. Joshi M, Akhtar M, Najmi AK, Khuroo AH, Goswami D. Effect of zinc in animal models of anxiety, depression and psychosis. Hum Exp Toxicol. 2012;31:1237-43

56. Connor MA, Chavkin C. Ionic zinc may function as an endogenous ligand for the haloperidol-sensitive sigma 2 receptor in rat brain. Mol Pharmacol. 1992:42:471-9.

57. Carrasco J, Penkowa M, Hadberg H, Molinero A, Hidalgo J. Enhanced seizures and hippocampal neurodegeneration following kainic acid-induced seizures in metallothionein-I + I-deficient mice. Eur J Neurosci. 2000;12:2311-22.

58. Kandratavicius L, Hallak JE, Carlotti CG, Assirati JA, Leite JP. Neurotrophin receptors expression in mesial temporal lobe epilepsy with and without psychiatric comorbidities and their relation with seizure type and surgical outcome. Acta Neuropathol Commun. 2014;2:81-98.

59. Kandratavicius L, Hallak JE, Carlotti CG, Assirati JA, Leite JP. Hippocampal expression of heat shock proteins in mesial temporal lobe epilepsy with psychiatric comorbidities and their relation to seizure outcome. Epilepsia. 2014;55:1834-43.

60. Ohnishi M, Monda A, Takemoto R, Fujimoto Y, Sugitani M, Iwamura T, et al. High-mobility group box 1 up-regulates aquaporin 4 expression via microglia-astrocyte interaction. Neurochem Int. 2014;75:32-8.

61. Scharfman HE, Binder DK. Aquaporin-4 water channels and synaptic plasticity in the hippocampus. Neurochem Int. 2013:63:702-11.

62. Skucas VA, Mathews IB, Yang J, Cheng Q, Treister A, Duffy AM, et al. Impairment of select forms of spatial memory and neurotrophin-dependent synaptic plasticity by deletion of glial aquaporin-4. J Neurosci. 2011;31:6392-7

63. Zhang Z, Fan J, Ren $Y$, Zhou W, Yin G. The release of glutamate from cortical neurons regulated by BDNF via the TrkB/Src/PLC-gamma1 pathway. J Cell Biochem. 2013;114:144-51.

64. Amiry-Moghaddam M, Williamson A, Palomba M, Eid T, de Lanerolle NC, Nagelhus EA, et al. Delayed K+ clearance associated with aquaporin-4 mislocalization: phenotypic defects in brains of alpha-syntrophin-null mice. Proc Natl Acad Sci U S A. 2003;100:13615-20.

65. Krogias C, Hoepner R, Muller A, Schneider-Gold C, Schroder A, Gold R. Successful treatment of anti-Caspr2 syndrome by interleukin 6 receptor blockade through tocilizumab. JAMA Neurol. 2013;70:1056-9. 
66. Chaudhry IB, Hallak J, Husain N, Minhas F, Stirling J, Richardson P, et al. Minocycline benefits negative symptoms in early schizophrenia: a randomised double-blind placebo-controlled clinical trial in patients on standard treatment. J Psychopharmacol. 2012;26:1185-93.

67. Muller N, Schwarz MJ, Dehning S, Douhe A, Cerovecki A, Goldstein-Muller B, et al. The cyclooxygenase-2 inhibitor celecoxib has therapeutic effects in major depression: results of a double-blind, randomized, placebo controlled, add-on pilot study to reboxetine. Mol Psychiatry. 2006:11:680-4.

68. Devinsky O, Vezzani A, Najjar S, De Lanerolle NC, Rogawski MA. Glia and epilepsy: excitability and inflammation. Trends Neurosci. 2013;36:174-84

\section{Submit your next manuscript to BioMed Central} and take full advantage of:

- Convenient online submission

- Thorough peer review

- No space constraints or color figure charges

- Immediate publication on acceptance

- Inclusion in PubMed, CAS, Scopus and Google Scholar

- Research which is freely available for redistribution 